\title{
Two-component Dark Matter in the vectorlike hypercolor exten- sion of the Standard Model
}

\author{
Vitaly Beylin ${ }^{1, \star}$, Maxim Bezuglov ${ }^{2,3, \star \star}$ and Vladimir Kuksa ${ }^{1, \star \star \star}$ \\ ${ }^{1}$ Research Institute of Physics, Southern Federal University, Pr. Stachky 194, Rostov-on-Don 344090, Rus- \\ sia \\ ${ }^{2}$ Bogoliubov Laboratory of Theoretical Physics, Joint Institute for Nuclear Research, Joliot-Curie 6, 141980 \\ Dubna, Moscow region, Russia \\ ${ }^{3}$ Moscow Institute of Physics and Technology (State University), 9 Institutskiy per., 141701 Dolgoprudny, \\ Moscow Region, Russian Federation
}

\begin{abstract}
We consider a minimal vectorlike extension of the Standard Model that naturally contains two types of stable neutral particles. They can be interpreted as the Dark Matter candidates. Here, the SM is supplemented by a new fermion (H-quark) sector that is in confinement providing by the $S U(2)_{H C}$ gauge group. H-quarks interact with the SM particles via standard electroweak bosons. In analogy with the conventional QCD, $\mathrm{H}$-quarks can form bound states, $\mathrm{H}$-hadrons, that emerge in the $\sigma$ - model framework. Along with the stable neutral H-pion, there is one more pseudo-goldstone (diquark) state, B0, which is stable and also can be a component of the DM. Mass splittings both for components of the H-pion triplet and for neutral H-pion and B0 have been calculated, it is shown that this splittings can be small. The relic abundance for this composition of the DM is analyzed as well as the DM particles scattering off nucleons. A feature of this scenario is that Dark Matter turns out to consist of particles which are close in mass but have different origin and interact differently with ordinary matter. So, the model predicts two-component structure of the DM together with some specific manifestations of it.
\end{abstract}

\section{Introduction}

At the moment, the DM existence is a firmly established fact, which is confirmed in many astrophysical observations. Dark Matter occupies about a quarter of the Universe mass and plays a crucial role in the evolution of galaxies. Despite the fact that the existence of this substance has been known for an almost a century and that it literally surrounds us, we still do not have the faintest idea what it is. The most popular explanation for DM phenomenon is considered to be the Weakly Interacting Massive Particles(WIMPs) and they will be discussed in this work. In addition to the DM, there are still some unexplained anomalies in the cosmic rays, for example see[1-3]. The source of this anomalies is unknown and widely discussed in literature. One possible explanation can be complex structure of the DM having more then one component, (for information about such models, see) [4-14] and

\footnotetext{
^e-mail: vitbeylin@gmail.com

$\star \star$ e-mail: bezuglov.ma@phystech.edu

$\star \star \star$ e-mail: vkuksa47@mail.ru
} 
references therein. In this work we will consider a vectorlike hyper-quark model with two-component structure of the DM.

\section{The model}

In this section, we briefly describe the model under consideration, for more details see [15-18].

In addition to a set of ordinary particles of the SM, the model contains a new fermion sector in confinement under the gauge group $S U(2)_{H C}$. To ensure interaction with the $\mathrm{SM}$, an additional group $S U(2)_{W}$ corresponding to the electroweak interaction is introduced. Thus, the extended model includes a doublet of Dirac H-quarks with zero hypercharge, which provides cancellation of anomalies.

Masses of hyper-fermions (H-quarks) arise due to a spontaneous symmetry breaking of a new singlet scalar field, H-sigma, which is mixed with the standard Higgs boson. The mixing angle plays very important role in the theory and it should be small enough that there was no conflict with the SM precision data. In other words, corresponding parameters of Peskin-Tackeuchi satisfy the necessary experimental restrictions $[17,18]$.

In analogy with low-energy quark meson models, H-quarks can form H-hadrons. Effective Lagrangian is constructed taking into account violation of the global SU(4) symmetry, which generate pseudo-goldstone modes. Analogously to linear sigma-model, the model contains a triplet of pseudoscalar H-pions $\left(\tilde{\pi}^{+}, \tilde{\pi}^{0}, \tilde{\pi}^{-}\right)$and (also) neutral H-baryon along with its antiparticle $\left(B^{0}, \bar{B}^{0}\right)$. It is important to note, that masses of these particles are equal at the tree level. H-baryon possess an additive conserving quantum number and $\mathrm{H}$-pions posses a multiplicative conserving quantum number. So, the model suggested naturally contains at least two stable particles, both of them can be considered as the DM components.

To consider the model in the DM aspect, only four parameters are needed. Firstly, it is the mass of H-pions and H-baryons at the tree $\operatorname{level}\left(m_{\tilde{\pi}}\right)$. Then, the mass of $\mathrm{H}$-sigma $\left(M_{\sigma}\right)$ and its vacuum expectation value $(u)$. All these parameters are assumed to be of the same order of magnitude about $1 \mathrm{TeV}$. The mixing angle $(\theta)$ between $\mathrm{H}$-sigma and the Higgs boson is the most important parameter here. Its value controls the matching of the model predictions with the Standard Model precision measurements. In order to avoid contradiction with the collider data, it is necessary that the value $S_{\theta} \equiv \sin \theta \lesssim 0.1$, as it follows from the calculations.

Further, another important model parameters are the mass splittings between H-baryons and neutral H-pions along with the mass difference for $\mathrm{H}$-pion triplet components. These splittings at oneloop level are caused by electroweak interaction and can be calculated directly. The results are as following: ${ }^{1} \frac{\left|m_{\tilde{\pi}}-M_{B}\right|}{m_{\tilde{\pi}}} \lesssim 0.03, \quad m_{\tilde{\pi}^{ \pm}}-m_{\tilde{\pi}^{0}} \approx 163 \mathrm{MeV}$ Thus, we have three stable particles which can constitute the Dark Matter: H-baryon along with its antiparticle (their properties are similar, so we consider them as the one component) and neutral H-pion which is the lightest in H-pion triplet.

\section{Interaction with the ordinary matter}

One of the main properties of the Dark Matter is its weak interaction with the ordinary matter. The best experimental restrictions for corresponding cross-sections were obtained by XENON[19] and LUX[20]. Any theory that analyze the Dark Matter structure and manifestations should not conflict with them.

\footnotetext{
${ }^{1}$ Note, the mass splitting between $m_{\tilde{\pi}}$ and $M_{B}$ depends on the parameter $\mu$ which is interpreted as a regularization scale in $\overline{M S}$ scheme. The value of $\mu$ can be reasonably chosen at the hypercolor scale $(200-1000) \mathrm{GeV}$. Then, as it follows from numerical analysis, it does not affect on any conclusions made in this article.
} 


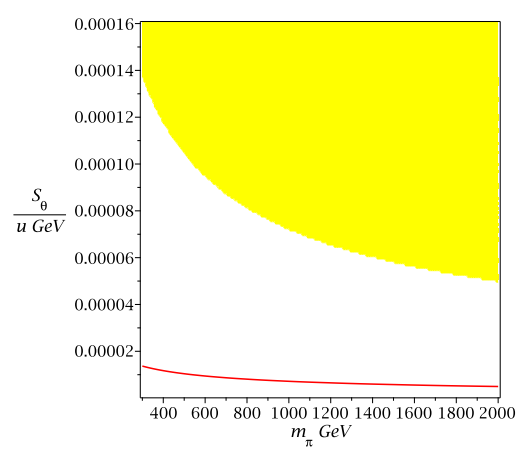

Figure 1. Phase diagram in terms of $S_{\theta} / u$ and $m_{\tilde{\pi}}$ parameters, the yellow regions is forbidden from direct experiments of the XENON and LUX collaborations. All regions above the red line will be excluded by future experiments in the next ten yeas[21].

At the tree level, the cross section for the H-color Dark Matter scattering off nucleons is described by the following simple formula:

$$
\sigma_{B N} \approx 3 * 10^{-43}\left(\frac{S_{\theta} M_{B}}{u}\right)^{2} \mathrm{~cm}^{2}, \quad M_{H}^{2} \ll M_{\sigma}^{2}, M_{B}^{2}, \quad C_{\theta} \approx 1
$$

Where $M_{H}$ is the mass of the Higgs boson. (see also Fig.1 for more details). Thus, there is a large range of parameters in which the model does not contradict the experimental data.

\section{Relic abundance in the two-component DM model}

Considering the relic density of $\mathrm{H}$-color particles, we will be interested not only in their total density but also in the ratio of various components of the DM. Relic density of the DM is determined by the Boltzmann kinetic equations. In this case, we need to write down five equations for each component. Fortunately, the situation can be easily simplified. Note that all charged H-pions will eventually decay into neutral one, therefore, we will only be interested in the total density of all $\mathrm{H}$-pions components together. To do this, it is necessary to take into account so-called co-annihilation processes[22] describing annihilation of all triplet components to each other. In the case of H-baryons analysis is analogous and even more simple. Then, instead of five equations we have only two:

$$
\begin{gathered}
\frac{d a^{3} n_{\pi}}{a^{3} d t}=<\overline{\sigma v}>_{\pi}\left(n_{\pi}^{2}-\left(n_{\pi}^{e q}\right)^{2}\right)-<\sigma v>_{\pi \pi}\left(n_{\pi}^{2}-\frac{9}{4} n_{B}^{2}\right)+<\sigma v>_{B B}\left(n_{B}^{2}-\frac{4}{9} n_{\pi}^{2}\right) \\
\frac{d a^{3} n_{B}}{a^{3} d t}=<\overline{\sigma v}>_{B}\left(n_{B}^{2}-\left(n_{B}^{e q}\right)^{2}\right)+<\sigma v>_{\pi \pi}\left(n_{\pi}^{2}-\frac{9}{4} n_{B}^{2}\right)-<\sigma v>_{B B}\left(n_{B}^{2}-\frac{4}{9} n_{\pi}^{2}\right),
\end{gathered}
$$


where:

$$
\begin{array}{r}
<\overline{\sigma v}>_{\pi}=\frac{1}{9} \sum_{i, j}<\sigma v>(i j \rightarrow X Y), \quad i, j=\tilde{\pi}^{+}, \tilde{\pi}^{-}, \tilde{\pi}^{0} ; \\
<\bar{\sigma} v>_{B}=\frac{1}{4} \sum_{\mu, v}<\sigma v>(\mu v \rightarrow X Y), \quad \mu, v=B \bar{B} \\
<\sigma v>_{\pi \pi}=\frac{1}{9}\left(<\sigma v>\left(\tilde{\pi}^{0} \tilde{\pi}^{0} \rightarrow B \bar{B}\right)+2<\sigma v>\left(\tilde{\pi}^{+} \tilde{\pi}^{-} \rightarrow B \bar{B}\right)\right) \\
<\sigma v>_{B B}=\frac{1}{2}\left(<\sigma v>\left(B \bar{B} \rightarrow \tilde{\pi}^{0} \tilde{\pi}^{0}\right)+<\sigma v>\left(B \bar{B} \rightarrow \tilde{\pi}^{-} \tilde{\pi}^{+}\right)\right)
\end{array}
$$

In addition to co-annihilations(4,5), analysis shows that processes mixing two components of the H-color Dark Matter with each other $(6,7)$ are also very important for the DM relic formation.

We solved the above kinetic equations numerically. The results are presented in the Fig.2 as the set of regions in the parameter plane-of $\mathrm{H}$-pion and $\mathrm{H}$-sigma masses. Here, dark blue color denotes areas in which we have the correct relic density of the H-color Dark Matter, in these regions fraction of H-pions is less than 25 percents. For red area everything is exactly the same, but here H-pions make up just over a quarter of the relic. We do not have any areas where H-pion component can dominate in the Dark Matter.

Further, blue shadowed areas correspond to permitted regions, but here we can not explain the Dark Matter relic abundance only by H-color components, and the yellow shadowed regions are forbidden by direct experiments of the XENON collaboration.

Thus, we have proved that the minimal vectorlike hypercolor extension of the Standard Model does not contradict the experimental data and also can serve as a basis for possible interpretation of the Dark Matter phenomenon.

\section{Conclusions}

We consider the simplest vectorlike hypercolor extension of the SM with one H-quark doublet and zero hypercharge that allows to cancel contributions of anomalies. The model includes a set of pseudogoldstone bosons among which there are two neutral stable states with close masses. If these particles are interpreted as the DM candidates, the model does not contradict current experimental data on the DM relic abundance and satisfies the constraints of LUX and XENON. Relative concentrations of these DM components were studied in detail. The appearance of two possible types of the DM carriers is a consequence of the structure and symmetry group of the considered minimal SM extension. Analysis of specific annihilation signals and deep inelastic scattering of high-energy cosmic rays off the DM particles is in progress.

\section{Acknowledgements}

M. Bezuglov would like to thank A.I.Onishchenko for interesting and stimulating discussions. This work is supported by RSCF Grant \# 18-12-00213.

\section{References}

[1] Nico Cappelluti et al., ApJ 854179 (2018)

[2] Daylan Tansu, et al., Physics of the Dark Universe 12 (2016): 1-23. 

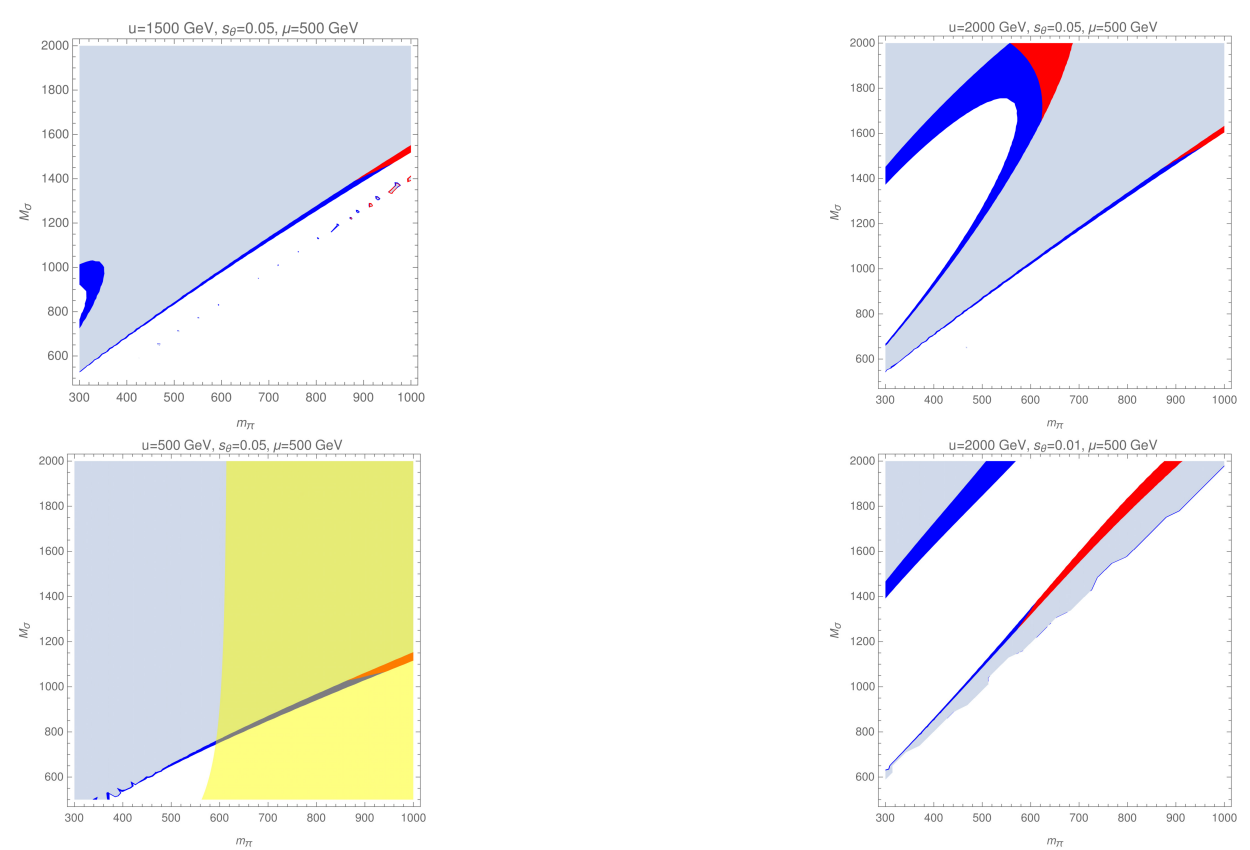

Figure 2. Results of numerical solution of kinetic equations(2,3) in a phase diagram in terms of $M_{\tilde{\sigma}}$ and $m_{\tilde{\pi}}$ parameters, for details on color codes see the text. For details on plot parameters see section 2.

[3] M. Aguilar et al., (AMS Collaboration) Phys. Rev. Lett. 117, 091103

[4] Dutta Banik, A., Pandey, M., Majumdar, D. et al., Eur. Phys. J. C (2017) 77: 657.

[5] A. Dutta Banik, M. Pandey, D. Majumdar, A. Biswas, Eur.Phys.J. C 77 (2017) no.10, 657

[6] Arcadi, G., Gross, C., Lebedev, O. et al., JHEP. (2016) 2016: 81.

[7] S. Esch, M. Klasen, C. E. Yaguna, JHEP 1409 (2014) 108

[8] J. Buch, P. Ralegankar, V. Rentala, JCAP 1710 (2017) no.10, 028

[9] M. Aoki, D. Kaneko, J. Kubo, Front.in Phys. 5 (2017) 53

[10] M. Aoki, T. Toma, JCAP 1701 (2017) no.01, 042

[11] A. Biswas, J.Phys. G 43 (2016) no.5, 055201

[12] A. Biswas, D. Majumdar, A. Sil, P. Bhattacharjee, JCAP 1312 (2013) 049

[13] M. Klasen, F. Lyonnet, F. S. Queiroz, Eur.Phys.J. C77 (2017) no.5, 348

[14] S. Bhattacharya, A. Drozd, B. Grzadkowski, J. Wudka, JHEP 1310 (2013) 158

[15] Beylin, V.; Bezuglov, M.; Kuksa, V.; Volchanskiy, N., Adv. in High Energy Phys., vol. 2017, 1765340.

[16] V. Beylin, M. Bezuglov, and V. Kuksa, Int. J. Mod. Phys. A 32, 1750042 (2017).

[17] R. Pasechnik, V. Beylin, V. Kuksa, G. Vereshkov, Phys. Rev. D 88, 075009 (2013).

[18] R. Pasechnik, V. Beylin, V. Kuksa, G. Vereshkov, Int.J.Mod.Phys. A 31, 1650036 (2016).

[19] E. Aprile et al. (XENON Collaboration), Phys.Rev.Lett. 119, 181301 (2017).

[20] D. S. Akerib et al. (LUX Collaboration), Phys.Rev.Lett. 116, 161301 (2016).

[21] J. Aalbers et al. (DARWIN Collaboration), JCAP 1611, no.11, 017 (2016).

[22] Kim Griest and David Seckel, Phys. Lett. D 43,(1991) 3191-3203. 